\title{
Ein Übel kommt selten allein
}

\author{
Typ-1-Diabetes ist als Autoimmungeschehen oft mit anderen autoimmunologischen Erkrankungen \\ der Schilddrüse, des Magen-Darm-Trakts oder der Nebennieren assoziiert. Nicht immer wird ein \\ Screening empfohlen. Daran denken sollte man bei schwer erklärbaren Hyper- oder Hypoglykämien.
}

\begin{abstract}
Die pathogenetische Endstrecke ist bei allen Autoimmunerkrankungen (AE) dieselbe: Es besteht ein Pool an selbstaffinen T-Zellen, und die Interaktion zwischen diesen T-Lymphozyten und den antigenpräsentierenden Zellen wird durch Umweltfaktoren peripher aktiviert. Dazu kommt, dass viele AE einen ähnlichen genetischen Hintergrund haben, d.h. die immunologische Synapse ist ähnlich. „Daraus ergibt sich, dass Patienten mit Typ-1-Diabetes ein höheres Erkrankungsrisiko für weitere Autoimmunerkrankungen haben", sagte Dr. Heinrich Kahles von der Medizinischen Universitätsklinik in Frankfurt/Main. Dazu gehören die Hashimoto-Thyreoiditis, M. Basedow, Zöliakie, Autoimmungastropathie und der M. Addison. Es stelle sich die Frage, ob und wann danach gefahndet werden solle, zumal diese endokrinen Erkrankungen oft die Stoffwechseleinstellung erschweren, so Kahles.
\end{abstract}

\section{Hashimoto-Thyreoiditis}

Die Prävalenz der Hashimoto-Thyreoiditis bei Typ-1-Diabetikern liegt bei 10-20\%. Dabei können Auto-Antikörper gegen Thyreoglobulin und Thyroxin-Peroxidase (TPO) nachgewiesen werden. Der entscheidende genetische Risikomarker ist HLA DQA1. „Doch nicht jeder Diabetiker mit entsprechendem Antikörperbefund erkrankt wirklich", so Kahles. Einen hohen prädiktiven Wert haben diese Antikörper aber dann, wenn diese bereits im ersten Jahr nach der Erstdiagnose des Diabetes nachgewiesen werden. In der subklinischen Phase kommt es schon häufiger zu Hypoglykämien. Ein Screening mit Bestimmung von TSH und Anti-TPO-Antikörpern empfiehlt sich bei der Diagnosestellung des Diabetes und dann alle 1-2 Jahre. Bei positivem Antikörperbefund sollte das TSH aber alle 6 Monate kontrolliert werden.

\section{Morbus Basedow}

Die Prävalenz des M. Basedow ist mit 3-6\% niedriger. Diagnoseweisend ist der Nachweis von Antikörpern gegen den TSH-Rezeptor (TRAK). Klinisch manifestiert sich die Erkrankung mit den klassischen Symptomen einer Hyperthyreose mit Tachykardie und Gewichtsverlust, auch finden sich meist ein Exophthalmus und eine Struma. Bzgl. des Diabetes kommt es zu steigender Insulinresistenz mit hyperglykämen Entgleisungen.

\section{Zöliakie}

Bei unerklärlichen Blutzuckerschwankungen sollte man immer an eine Malabsorption als Folge einer Zöliakie denken, auch wenn ansonsten die typische Symptomatik wie Durchfälle fehlt. Die Prävalenz bei Typ-1-Diabetikern beträgt 1-9\%. Im Labor finden sich IgA-Antikörper gegen Gewebstransglutaminase (tTG2) und Endomysium. Diese Antikörper und auch die genetischen HLARisikomarker (HLA-DQ2 und HLA-DQ8) können zur Ausschlussdiagnostik verwendet werden. Die Diagnose sollte immer durch Dünndarmbiopsien bestätigt werden.

\section{Autoimmungastropathie}

Bei dieser AE werden Antikörper gegen Parietalzellen des Magens gebildet. Solche finden sich bei ca. 20\% der Typ-1-Diabetiker, doch nur jeder Fünfte mit entsprechendem Antikörperbefund erkrankt daran. Klinisch kommt es zu einer Hypo- bzw. Achlorhydrie mit konsekutiver Hypergastrinämie, woraus sich in Einzelfällen ein Karzinoid entwickeln kann. Auch kann ein Intrinsic-Faktor-Mangel, sprich eine perniziöse Anämie auftreten. Auf den Blutzucker hat die Erkrankung keine Auswirkungen.

\section{Morbus Addison}

Dies ist die seltenste Ko-AE, die sich aber in Kombination mit anderen AE als genetisch determiniertes Autoimmun-Polyglanduläres Syndrom (APS) manifestieren kann. Pathogenetisch relevant sind Autoantikörper gegen 21-Hydrolase. 30-40\% der antikörperpositiven Diabetiker erkranken. Wegen der sehr niedrigen Prävalenz wird ein Screening nicht empfohlen.

\section{Prävention mit Vitamin D?}

Die Frage, ob ein Vitamin-D-Mangel eine Rolle bei der Manifestation von Autoimmunerkrankungen spielen könnte, wird seit vielen Jahren sehr kontrovers diskutiert. „Bisher gibt es aber keinen Belege für eine präventive Wirkung“, so Kahles. Eine hochdosierte Supplementierung außerhalb von Studien könne deshalb nicht empfohlen werden. Ein Vitamin D-Mangel sollte aber unbedingt vermieden werden.

Dr. med. Peter Stiefelhagen

\section{Quelle: 51. DDG-Jahrestagung, 05.05.2016 in Berlin}

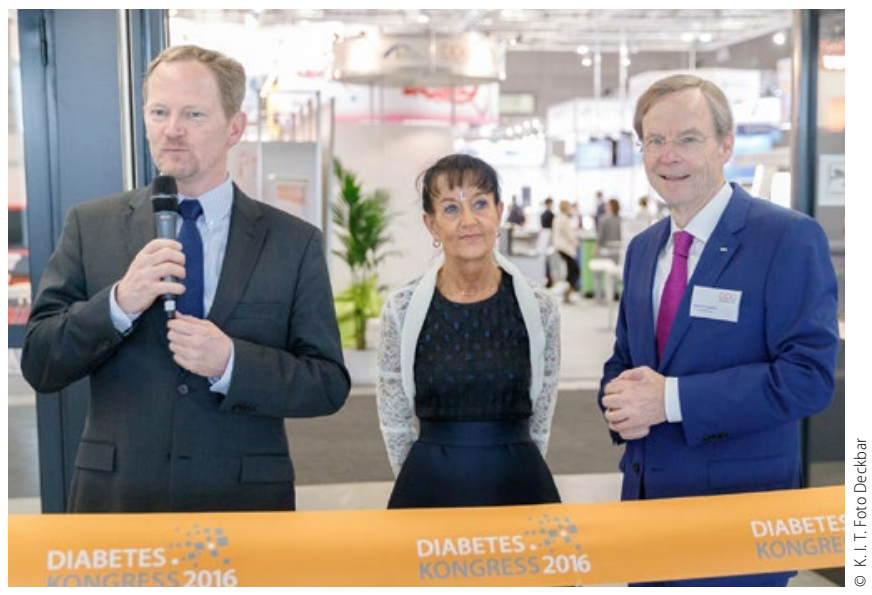

Von links nach rechts: Prof. Andreas Hamann (Kongresspräsident Diabeteskongress 2016), Siegrid Hoffmann (Unternehmen AstraZeneca) und Dr. Dietrich Garlichs (Geschäftsführer Deutsche Diabetes-Gesellschaft) bei der Eröffnung der Industrieausstellung. 